\title{
Glutaredoxin Desensitizes Lens to Oxidative Stress by Connecting and Integrating Specific Signaling and Transcriptional Regulation for Antioxidant Response
}

\author{
Qi Fan ${ }^{a, b}$ Yonglong Zhang ${ }^{c}$ Yanfeng Liud ${ }^{d, e}$ Jin Yang ${ }^{a, b}$ Dan Li ${ }^{a, b}$ Yating Tanga,b \\ Lei Caia,b Zhennan Zhao ${ }^{a, b}$ Peng Zhou ${ }^{f}$ Yi Lu $^{a, b}$ \\ aDepartment of Ophthalmology, Eye \& ENT Hospital of Fudan University, ${ }^{\mathrm{b}}$ Myopia Key Laboratory \\ of Health PR China, 'Department of Biliary-Pancreatic Surgery, Renji Hospital, School of Medicine, \\ Shanghai Jiao Tong University, ${ }^{\mathrm{d} D e p a r t m e n t}$ of Surgery, Huashan Hospital, Fudan University, ${ }^{\text {KKey }}$ \\ Laboratory of Medical Molecular Virology (MOE \& MOH), Shanghai Medical College, Fudan University, \\ 'Department of Ophthalmology, Parkway Health Hong Qiao Medical Center, Shanghai, China
}

\section{Key Words}

Cataract • Glutaredoxin • Antioxidant Response • SILAC

\begin{abstract}
Background/Aims: Oxidative stress plays a critical role in the development of cataracts, and glutaredoxins (Grxs) play a major protective role against oxidative stress in the lens. This study aimed to reveal the global regulatory network of Grx1. Methods: Stable isotope labeling by amino acids in cell culture (SILAC) was used in a proteome-wide quantitative approach to identify the Grx1 regulatory signaling cascades at a subcellular resolution in response to oxidative stress. Results: A total of 1,291 proteins were identified to be differentially expressed, which were further categorized into a variety of signaling cascades including redox regulation, apoptosis, cell cycle control, glucose metabolism, protein synthesis, DNA damage response, protein folding, proteasome and others. Thirteen key signaling node molecules representing each pathway were verified. Notably, the subunits of proteasome complexes, which play a pivotal role in preventing cytotoxicity via the degradation of oxidized proteins, were highly enriched by Grx1. By data-dependent network analysis, we found global functional links among these signaling pathways which elucidate how Grx1 integrates the operation of these regulatory networks in an interconnected way for $\mathrm{H}_{2} \mathrm{O}_{2}$-induced response. Conclusion: Our data provide a system-wide insight into the function of Grx1 and provide a basis for further mechanistic investigation of Grx1 in antioxidant responses in the lens.

Q. Fan, Y. Zhang and Y. Liu contributed equally to this work. 


\section{Introduction}

Cataracts, which are characterized by lens opacity and visual impairment [1], are a major cause of blindness worldwide [2,3]. By the year 2020, the number of cataract with reduced vision of $3 / 60$ or worse will have increased to 40 million [4]. Currently, the main therapy for cataracts is surgery. A delay of 10 years in the onset of cataracts would decrease the number of individuals requiring surgery by $45 \%$ [5]. Therefore, how to prevent and/or delay the onset of cataracts has been widely investigated.

Oxidative stress plays a major role in the pathogenesis of cataracts [6]. Lens contain a high concentration of thiol groups that must be in the reduced state to maintain the clarity of the lens. When the lens is exposed to oxidative stress, high local oxygen concentrations, such as reactive oxygen species (ROS), can be produced [7]. ROS attack thiol groups in proteins and induce disulfide bridges between proteins and thiol containing small molecular compounds such as glutathione, leading to the formation of S-glutathionylated proteins (PSSGs). PSSGs induce a cascade of events, including protein and/or enzyme deactivation, alterations of protein conformation, protein-protein aggregation, opacification of the lens and the eventual development of a cataract [8]. Therefore, maintaining the redox status of lens proteins is critical for maintaining lens transparency.

Glutaredoxins (Grxs) have been identified as thioltransferases (TTases), which catalyze thiol/disulfide exchange oxidoreduction reactions, resulting in the reversible reduction of protein-glutathionyl mixed disulfides to free sulfhydryl groups through a monothiol mechanism [9]. The Grx family has two major subsets: cytosolic (Grx1) and mitochondrial (Grx2) enzymes [10]. Grx1 belongs to the classical Grx group of enzymes, which are present in the cytosol and the mitochondrial intermembrane space [11, 12]. It is an $11.8-\mathrm{kDa}$ protein containing a conserved CXXC sequence at the active site [13]. It has been shown that Grx1 regulates various aspects of cellular function; for example, it is involved in cellular differentiation [14], transcription [15], DNA replication [16], protection against oxidative stress [17] and apoptosis signaling [18]. Li S reported that Grx1 can alleviate oxidative stress and apoptotic damage in coronary artery endothelial cells induced by high glucose concentrations [19], and Cater MA proved that Grx1 can protect neuronal cells from copperinduced toxicity [20]. Grx2 is 34\% identical to the Grx1 and is localized to the mitochondrial matrix. It plays an important role as a redox sensor in the mitochondria as an iron-sulfur protein [21]. It can prevent apoptosis by preventing cytochrome c release [22] and protecting complex I activity in the mitochondria [23].

Grx can protect the lens against oxidative stress. Grx dethiolates PSSG, maintains thiol groups in the reduced state in the lens. Under ultraviolet radiation (UVR) exposure, TTase activity and protein expression have been shown to be strongly upregulated in the lenses of young mice [24]. TTase ${ }^{-/}$lens epithelial cells are less viable and show greater rates of apoptosis, and they are less efficient at removing $\mathrm{H}_{2} \mathrm{O}_{2}$ than TTase ${ }^{+/+}$cells, and introducing purified TTase into TTase $\%$ cells restores antioxidant functions [10]. In addition, the Grx1 gene provides a $44 \%$ increase of protection against close to threshold UVR-B induced oxidative stress compared to the absence of the Grx1 gene [25]. An intact Grx1 gene also allows cells to tolerate a 1.3-times longer exposure to the oxidative stress induced by $300 \mathrm{~nm}$ UVR than is observed in cells where the Grx1 gene is absent [26].

However, the mechanistic details of the antioxidant function of Grx have not been fully described. Previous studies have shown that Grx bind to apoptosis signal-regulating kinase 1 (ASK1) and suppress its activation [27]. Chantzoura E discovered a crucial role for Grx1 in the TRAF6-dependent activation of NF- $\mathrm{KB}$ via IL-1R/TLRs [28], and Liu et al. demonstrated that by preventing AKT glutathionylation, Grx1 can protect retinal pigment epithelial (RPE) cells against oxidative stress-induced apoptosis [29]. However, these studies have not comprehensively elucidated the functions of Grx1 or the mechanisms by which they are regulated.

Stable isotope labeling by amino acids in cell culture (SILAC) is a powerful approach for performing a mass spectrometry (MS)-based proteome-wide quantitative identification of 
changes in protein abundance and/or protein modifications [30, 31]. In the present study, we used SILAC to investigate the functional links between Grx1 and putative target proteins involved in the multiple pathways and biological processes (BPs) responsible for antioxidant functions in the lens. The results will be helpful in the development of more precise and effective strategies for preventing the onset of cataracts.

\section{Materials and Methods}

\section{Reagent and Antibodies}

Dulbecco's modified Eagle medium (DMEM) and fetal bovine serum (FBS) were obtained from GIBCO (Life Technology, USA). Stable isotope-labeled amino acids, including Lys-8 (L-13C615N2-lysine) and Arg10 (L-13C615N4-arginine), were purchased from Cambridge Isotope Laboratories (UK). SILAC medium without L-arginine, L-glutamine, and L-lysine was purchased from SILACgen (Shanghai, China), and 30\% hydrogen peroxide $\left(\mathrm{H}_{2} \mathrm{O}_{2}\right)$ solution was obtained from Sigma-Aldrich (Shanghai, China). SYBR ${ }^{\circledR}$ Premix Ex $\mathrm{Taq}^{\mathrm{TM}} \mathrm{GC}$ was purchased from TaKaRa (Dalian, China).

Antibodies, including anti-Grx1, anti-SOD1, anti-SOD2, anti-BAD, anti-CAT, anti-VDAC, anti-HSP90B1, anti-CDC25C, anti-caveolin-1, anti-GSTM3, and anti-DDB1, were purchased from Proteintech (Wuhan, China). Antibodies against PKM2 and $\beta$-actin were obtained from Cell Signaling Technology (Danvers, MA, USA).

Plasmid Constructs and Quantitative Real-time PCR (qRT-PCR)

DNA fragments encoding Grx1 were introduced into pCMV-3×FLAG-7.1 at EcoRI and SalI sites. $3 \times$ FLAG-tagged Grx1 fragments were further cloned and inserted into pCDH-CMV-EF1-puro to generate the pCDH-CMV-FLAG-Grx1-EF1-puro vector. DNA fragments encoding the hairpin precursors for siGrx1 (5'-GCCGCTTGCACGTATAGATAC-3') were inserted into pLKO.1TRC. A scramble (Scr) sequence was used as a negative control.

For the qRT-PCR analysis, total RNA was extracted using TRIzol Reagent (Invitrogen), and reverse transcription was performed using a PrimeScript RT reagent kit (TaKaRa) according to the manufacturer's instructions. For the qRT-PCR analysis, aliquots of cDNA were amplified using SYBR Premix Ex Taq (TaKaRa). PCR reactions were performed in triplicate under the following conditions: $95{ }^{\circ} \mathrm{C} / 30 \mathrm{~s}, 40$ cycles of $95{ }^{\circ} \mathrm{C} / 5$ $\mathrm{s}, 60^{\circ} \mathrm{C} / 15 \mathrm{~s}$, and $72^{\circ} \mathrm{C} / 10 \mathrm{~s}$ in a Q1 MXP3000 cycler (Stratagene, USA), with each reaction repeated at least three times. The following primer pairs were used: Grx1: forward, 5'-GGA GCA AGA ACG GTG CCT CGA G-3'; reverse, 5'-AAA GCA GAT TGG AGC TCT GCA G-3'. SOD1: forward, 5'-CGA GCA GAA GGA AAG TAA TGG-3'; reverse, 5'-CAC TGG TAC AGC CTG CTG TAT T-3'. SOD2: forward, 5'-CGA CCT GCC CTA CGA CTA C-3'; reverse, 5'-TGA CCA CCA CCA TTG AAC TTC-3'. VDAC1: forward, 5'- CTC ACC TGA ATG GGA CTT T-3'; reverse, 5'-TCA CAT CCA CCT TCT CCA C-3'. PKM2: forward, 5'-GCC TGC TGT GTC GGA GAA G-3'; reverse, 5'-CAG ATG CCT TGC GGA TGA ATG-3'. Catalase: forward, 5'-CCA TTA TAA GAC TGA CCA GGG C-3'; reverse, 5'-AGT CCA GGA GGG GTA CTT TCC-3'. CDC25C: forward, 5’AAG TGG CCT ATA TCG CTC CC-3'; reverse, 5'- CCC TGG TTA GAA TCT TCC TCC A-3'. DDB1: forward, 5'-TGC GTG ACC GGA CAC TTT ACT-3'; reverse, 5'-GCC TGA AAA GCT CCA TGA CCG C-3'. HSP90B1: forward, 5'-GAG ATC AAA GAC TAC AGT CCC-3'; reverse, 5'-GTT CGT GCT CAT ACT TGG TC-3'. RUVBL1: forward, 5'-ACA AAC TTC GAG GGG AGA TT-3'; reverse, 5'-CGA TGG GAG CGA TAG AAG AC-3'. Caveolin-1: forward, 5'-CGT AGA CTC GGA GGG ACA TC-3'; reverse, 5'-TTT CGT CAC AGT GAA GGT GG3'. GSTM3: forward, 5'-ATA TTC GTG GGC TGG CGC AC-3'; reverse, 5'-TAG TCA GGA GCT TCC CCG CAC GT-3'.

\section{Cell Culture and Lentivirus Preparation}

An embryo kidney cell line (HEK293T) and human lens epithelial cells (SRA01/04) were obtained from the Cell Bank of the Shanghai Institutes of Biological Sciences, Chinese Academy of Sciences.

For lentiviral production, 293FT cells were prepared in a $10-\mathrm{cm}$ dish and grown to $30 \%$ confluence. They were co-transfected for $12 \mathrm{~h}$ with $10 \mu \mathrm{g}$ of the target plasmid (pCDH-CMV-FLAG-Grx1-EF1-puro or pLKO.1-siGrx1), $7.5 \mu \mathrm{g}$ of the psPAX2 packaging plasmid, and $2.5 \mu \mathrm{g}$ of the pMD2.G envelope plasmid using SimpleTrans reagent (SILACgen, Shanghai). Next, the transfection reagent was replaced with $15 \mathrm{~mL}$ of fresh medium, and the cells were incubated for $48 \mathrm{~h}$. The medium containing virus particles was collected via centrifugation at 1,250 rpm for $5 \mathrm{~min}$. For the selection of stable cell lines, the cells were infected with lentiviruses for $24 \mathrm{~h}$ and further selected with puromycin $(5 \mu \mathrm{g} / \mathrm{mL})$ for $48 \mathrm{~h}$. 
SILAC Labeling and SILAComics Identification of the Grx1 Regulatory Network

The methods used for the SILAC labeling and SILAComics identification have been described previously [32]. Briefly, for SILAC labeling, SRA01/04 cells were cultured in DFBS DMEM high-glucose medium (with L-glutamine and without L-arginine and L-lysine), supplemented with "heavy" amino acids (R10K8) and 10\% DFBS for nine passages. The incorporation efficiency was further confirmed via MALDITOF-MS. Approximately $1 \times 10^{7}$ of each type of "heavy"-labeled SRA01/04 cell infected with the lentivirus and normal SRA01/04 cells were treated with $100 \mu \mathrm{M} \mathrm{H}_{2} \mathrm{O}_{2}$ for $24 \mathrm{~h}$ and were then lysed and separated into their nuclear extracts and cytoplasmic fractions. Protein extracts were then quantified with a protein assay kit (BioRad) and equally mixed. Mixed cell lysates were separated via SDS-PAGE, followed by band excision, trypsin digestion, and LC-MS/MS identification and quantification.

\section{Western Bloting and Co-immunoprecipitation Assay}

Cells were washed with PBS for three times and lysed by WB\&IP lysis buffer (1\% Nonidet P-40, 150mM $\mathrm{NaCl}, 100 \mathrm{mM}$ Hepes, 5mM Na4P207, 5mM NaF, 2mM Na3V04)(SILACgen, Shanghai, China) supplemented with protease inhibitor mixture and phosphatase inhibitors(Roche Applied Science) for 30 minutes. The cell lysates were then centrifuged at $16000 \mathrm{~g}$ for 15 minutes. The supernatants were separated by SDS-PAGE and subjected for western blotting analysis. For immunoprecipitation assay, the supernatants were incubated with FLAG beads or anti-GSH and protein G(sigma, Germany) at $4^{\circ} \mathrm{C}$ for 3 hours followed by being washed five times with lysis buffer, and were then eluted by boiling in 1× SDS loading buffer for western blotting analysis.

\section{Statistical Analysis}

Student's t-tests were used to compare qualitative and quantitative variables. All statistical tests were two-sided, and a $P$-value of $<0.05$ was considered to indicate a statistically significant difference.

\section{Results}

Grx1 is Specifically Induced by Oxidative Stress

To uncover the function of Grx1 during cataract development caused by exposure to oxidative stress, we first determined whether Grx1 is expressed in the SRA01/04 human lens epithelial cell line. Using qRT-PCR and western blotting analyses, the mRNA transcript and protein levels of Grx1 were found to be only detectable in unperturbed cells, but were robustly induced after $24 \mathrm{~h}$ of $\mathrm{H}_{2} \mathrm{O}_{2}$ treatment (Fig. 1A). We also observed increased levels of Grx1 in response to elevated concentrations of $\mathrm{H}_{2} \mathrm{O}_{2}$ and prolonged incubation times (Fig. 1B and 1C), suggesting that Grx1 is constitutively expressed at very low levels and is specifically induced in a dose-dependent manner in response to oxidative stress. Additionally, oxidative stress appeared to re-activate Grx1 expression at the level of transcription. Unlike thioredoxin 1, which is controlled by the inhibitor thioredoxin-interacting protein (TXNIP), which tightly controls the cellular redox state, Grx1 has not yet been reported to have an endogenous repressor.

\section{SILAComics Identification of the Grx1 Regulation Network}

Although a few Grx substrates have been identified, the biological effects of Grx1 in response to oxidative stress in particular remain largely unknown. We performed a proteomewide identification of Grx1 target genes and/or proteins at both the transcriptional and translational level using a SILAC-based LC-MS/MS quantitative proteomic approach to identify differentially expressed proteins (DEPs) by comparing the protein profiles present when Grx1 was over-expressed or depleted following $\mathrm{H}_{2} \mathrm{O}_{2}$ treatment. Briefly, SRA01/04 cells were cultured in a "heavy" medium containing Lys-8 (L-13C615N2-lysine) and Arg10 (L-13C615N4-arginine) for at least five passages. Labeled SRA01/04 cells were further separated into their cytosolic and nuclear fractions, followed by an equal mixing of these cell fractions from Grx1-expressing cells or those with shRNA constructs. The resulting fractions were then separated via SDS-PAGE, and gel slices were subjected to tryptic digestion and peptide extraction, followed by a LC-MS/MS analysis performed in two replicates (Fig. 2A 
Fig. 1. Oxidative stress activates Grx1 expression in a dose- and time-dependent manner. (A) Grx1 is specifically induced by $\mathrm{H}_{2} \mathrm{O}_{2}$. Left, cells treated with $100 \mu \mathrm{M} \mathrm{H}_{2} \mathrm{O}_{2}$ for $24 \mathrm{~h}$ were lysed for western blot analysis using an anti-Grx1 antibody. Right, qRT-PCR analyses was used to measure Grx1 mRNA levels in the indicated cells. (B) Induction of Grx1 protein by oxidative attack is dependent on $\mathrm{H}_{2} \mathrm{O}_{2}$ concentration and incubation time. Left, cells incubated with the indicated concentrations of $\mathrm{H}_{2} \mathrm{O}_{2}$ for $12 \mathrm{~h}$ were lysed and analyzed via western blotting using an anti-Grx1 antibody. Right, cells incubated with the indicated concentrations for the indicated times were lysed and analyzed via western blotting using an anti-Grx1 antibody. (C) $\mathrm{H}_{2} \mathrm{O}_{2}$ activates Grx1 mRNA expression, which is dependent on $\mathrm{H}_{2} \mathrm{O}_{2}$ concentration and incubation time. Left, cells incubated with the indicated concentration of $\mathrm{H}_{2} \mathrm{O}_{2}$ for $12 \mathrm{~h}$ were analyzed via qRT-PCR analyses. Right, cells incubated with the indicated concentrations of $\mathrm{H}_{2} \mathrm{O}_{2}$ for the indicated times were analyzed via qRT-PCR. Representative results are shown with three independent experiments performed in triplicate. ${ }^{*} P<0.05$, ${ }^{* *} P<0.01,{ }^{* * *} P<0.001$. Student's-t tests were used for the statistical analysis.

and 2B). Based on previous criteria and the quality of MS data with a higher signal-to-noise ratio, a threshold of a $20 \%$ change in protein abundance $[33,34]$ (a light-to-heavy ratio of $>1.2$ or $<0.8$ ) was used to define whether a protein was differentially expressed. A total of 1,291 DEPs were identified with high confidence based on this threshold.

\section{Functional Categorization and Validation of Grx1-enriched Proteins}

Of the DEPs quantified in the two LC-MS/MS datasets, a total of 1,291 showed enrichment by Grx1 at high level of confidence. We then performed a gene ontology (GO) functional enrichment analysis using the Protein Analysis THrough Evolutionary Relationships (PANTHER; in the public domain, http://www.pantherdb.org/) and Database for Annotation, Visualization, and Integrated Discovery (DAVID; http://david.abcc.ncifcrf. gov/) online tools. Based on the known pathways, these DEPs were mainly categorized into a variety of signaling cascades, including redox regulation, apoptosis, cell cycle control, glucose metabolism, protein synthesis, DNA damage response, protein folding, proteasome, and others (Fig. 3A). In assessing subcellular localization, we found that mitochondrial proteins were highly enriched by Grx1 (Fig. 3B). Mitochondria are the major producers of intracellular ROS, which have been linked to age-related changes and various chronic diseases [35]. Growing evidence [36, 37] shows that mitochondria-targeted proteins could 


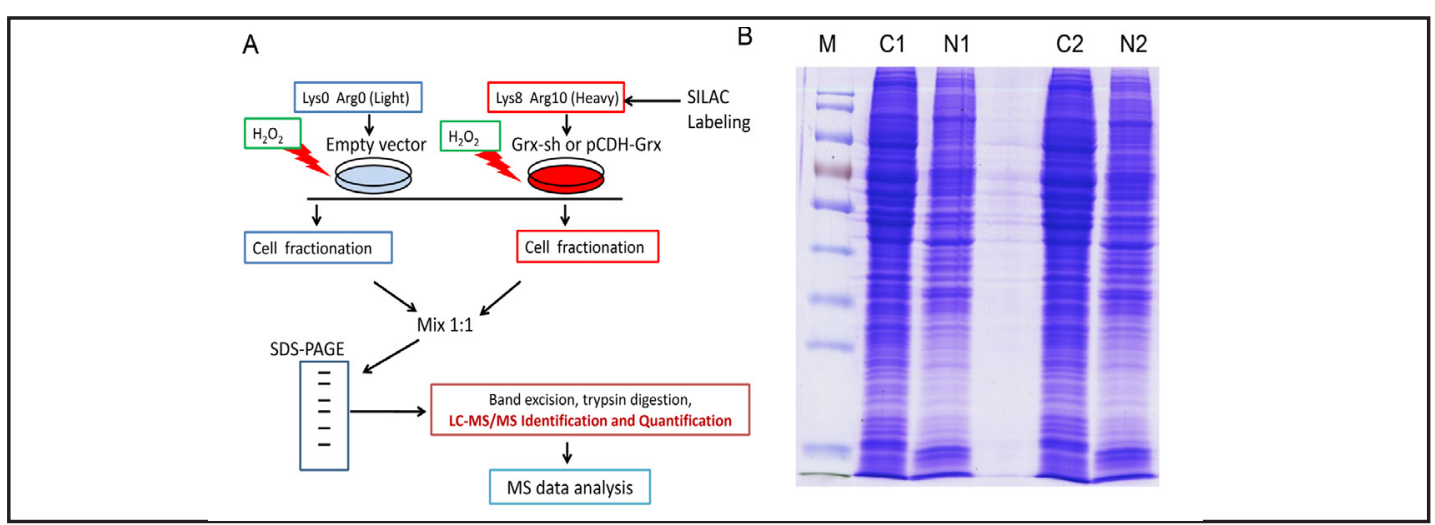

Fig. 2. SILAC-based proteome-wide identification of the Grx1 regulatory network in SRA01/04 cells. (A) Workflow of the SILAC-based quantitative proteomic approach. SRA01/04 cells were infected with lentiviruses and labeled with "heavy" amino acids. Labeled and normal SRA01/04 cells were lysed and separated into their cytoplasmic and nuclear fractions. The cytoplasmic fractions were equally mixed, as well as the nuclear fractions, followed by SDS-PAGE separation, in-gel digestion, and LC-MS/MS identification and quantification. (B) Coomassie blue staining of the cell lysate fractions. Equally mixed cell fractions were separated via SDS-PAGE followed by Coomassie blue staining. M, protein markers. C1, cytosolic fractions of Grx1-depleted and Scr control cells. N1, nuclear fractions of Grx1-depleted and Scr control cells. C2, cytosolic fractions of Grx1-expressing and vector control cells. N2, nuclear fractions of Grx1-expressing and vector control cells.

serve as potentially effective antioxidant therapeutic agents for use against the damage caused by enhanced ROS generation. These observations imply that Grx1 might be tightly linked to mitochondrial function in response to oxidative stimuli.

Enriched BP GO terms were grouped into 21 clusters $(P<0.01)$ (Fig. 3C); the top five clusters were metabolic process, respiratory electron transport chain, protein folding, translation, and transport. Eighteen clusters were found in the enriched molecular function (MF), with calcium-dependent phospholipid binding, NADH dehydrogenase, GDP binding, oxidoreductase activity, and syntaxin binding were mostly enriched $(P<0.01)$ (Fig. 3D). These findings indicate the functional diversity of Grx1 in response to oxidative stress. To verify the accuracy of the identification and quantification of our proteomic data, we performed western blotting analyses to validate selected target proteins representative of key signaling pathways (Fig. 4).

Grx1 Controls the Oxidative Stress-mediated Regulation of Proteasome Complexes

ROS-induced oxidative stress has been implicated in aging and many human diseases, notably in neurodegenerative disorders and age-related cataracts [38]. Oxidative stressors can chemically modify proteins, leading to protein oxidation, which can alter their biological function. Numerous studies have demonstrated that proteasome complexes play a pivotal role in preventing cytotoxicity via the selective recognition and degradation of oxidized proteins $[39,40]$. Despite the critical role of proteasome complexes in the oxidative stress response, our current understanding of how they are regulated is limited. Herein, we found 23 subunits of proteasome complexes that were significantly enriched by Grx1 $(P<$ 9.1 E-49). This suggests that Grx1 is involved in the regulation of proteasome complexes, which might subsequently attenuate oxidation-induced cytotoxicity through enhanced proteasome-dependent degradation of oxidized proteins. The functional links between Grx1 and proteasome complexes might provide a basis for developing new strategies for the possible treatment of age-related cataracts.

Grx1 Targets the Antioxidant Signaling Pathway for Redox Homeostasis

Redox homeostasis is maintained by the antioxidant defense system, which is responsible for eliminating a wide range of oxidants, including ROS. Increasing evidence [41, 


\section{Cellular Physiology Cell Physiol Biochem 2016;39:1813-1826 \begin{tabular}{ll|l} 
DOI: 10.1159/000447881 & $\begin{array}{l}\text { O 2016 The Author(s). Published by S. Karger AG, Basel } \\
\text { wwwwkarger.com/cpb }\end{array}$
\end{tabular}

Fig. 3. Functional categorization and distribution of Grx1-enriched proteins suggests Grx1 targets and integrates key signaling pathways for a systematic response to oxidative stress. (A) Signaling pathway categorization of Grx1-enriched proteins using DAVID reveals Grx1 crosstalk with redox regulation, apoptosis, cell cycle control, glucose metabolism, protein synthesis, DNA damage response, protein folding, and proteasome regulation. The lists of differentially expressed proteins were submitted to the online DAVID database and proteins were classified using a signaling pathway analysis. The most enriched signaling cascades are shown. (B) The subcellular localization of Grx1-enriched proteins suggests that Grx1 is ubiquitously involved in the compartment-specific regulation of its substrates. The lists of differentially expressed proteins were submitted to the online DAVID database and proteins were classified using a localization analysis. Percentages of the indicated subcellular localization are shown. (C) Biological process assignments of Grx1-regulated proteins using DAVID indicate that Grx1-regulated proteins are grouped into 21 clusters: the top five clusters were metabolic process, respiratory electron transport chain, protein folding, translation, and transport. (D) Molecular function assignments using DAVID show that Grx1-enriched proteins are mostly clustered into functions related to calcium-dependent phospholipid binding, NADH dehydrogenase, GDP binding, oxidoreductase activity, and syntaxin binding. Eighteen clusters were found and are shown here. $P<0.01$ indicates a significant difference.

42] indicates that the antioxidant defense system is incorporated into signaling pathways that determine the response of cells to disruptions in their physiological processes.

Superoxide dismutase (SOD) and catalaseare the major scavenging enzymes that facilitate the conversion of superoxides into hydrogen peroxide. Changes in either the expression or the localization of these enzymes are likely to modulate redox homeostasis and to regulate various signaling pathways $[43,44]$. Notably, catalase1, SOD1, and SOD2 were found to be significantly downregulated in Grx1-depleted cells and were elevated in Grx1-overexpression cells, suggesting that Grx1 directs the antioxidant response by upregulating key antioxidant defense enzymes. In addition, several members of the glutathione S-transferase (GST) group, a major group of detoxification enzymes (e.g., GSTM3), were found to be upregulated by Grx1 expression, indicating that Grx1 amplifies oxidative responses via a feedback cascade involving key antioxidant enzymes.

\section{Grx1 Directs Metabolic Remodeling in Response to Oxidative Stress}

Intracellular levels of ROS are vital for controlling many aspects of cell proliferation and survival. It has been proposed that the acute elevation of intracellular ROS levels results in the inhibition of glycolysis and the activation of the pentose phosphate pathway (PPP) as a firstline response to oxidative stress and that this is regulated by pyruvate kinase isozyme type M2 (PKM2) [45]. In the present study, we found that PKM2 is decreased by Grx1, which might 
Fig. 4. Grx1 regulates the expression of key signaling modules in human epithelial lens cells upon $\mathrm{H}_{2} \mathrm{O}_{2}$ stimulation. SRA01/04 cells infected with the indicated lentivirus were treated with $100 \mu \mathrm{M}$ $\mathrm{H}_{2} \mathrm{O}_{2}$ for $12 \mathrm{~h}$ and then lysed for SDS-PAGE separation and western blot analysis using the indicated antibodies. Key signaling molecules representing redox homeostasis, apoptosis, cell cycle control, glucose metabolism, DNA damage response, protein folding, and transcription activity were selected for verification.

Fig. 5. Grx1 selectively interacts and glutathionylates critical signaling proteins, altering protein stability. (A) Grx1 serves as a transcriptional regulator for the upregulation of SOD1, SOD2, CAT, RUVBL1, DDB1, and caveolin-1. SRA01/04 cells infected with the indicated lentivirus were treated with $100 \mu \mathrm{M} \mathrm{H}_{2} \mathrm{O}_{2}$ for $12 \mathrm{~h}$. RNA extraction and qRT-PCR analyses were performed to evaluate the mRNA transcripts. (B) Grx1 interacts with SOD1, SOD2, PKM2, CDC25C, HSP90B1 and VDAC1. SRA01/04 cells rus were lysed for immunoprecipitation using anti-flag beads, and the immunoprecipitates were detected using the indicated antibody. (C) Results indicate that SOD1, SOD2, PKM2, CDC25C, HSP90B1 and VDAC1 are

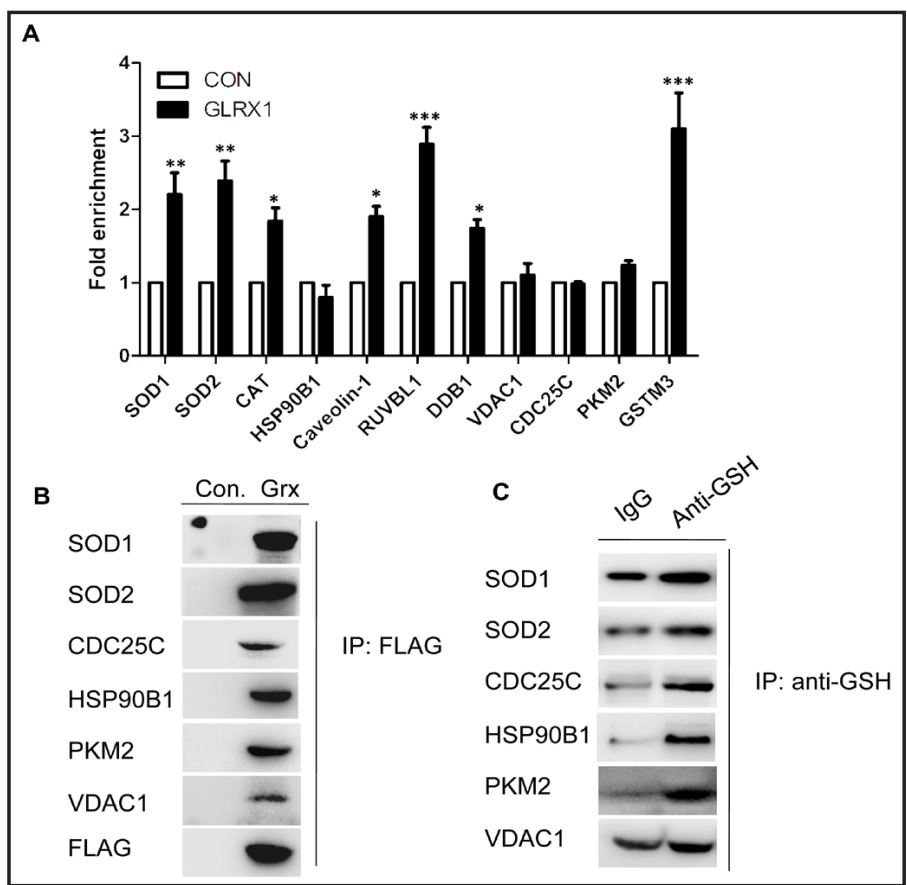
infected with the Flag-Grx1 lentivi-

glutathionylated in vitro. SRA01/04 cells treated with $100 \mu \mathrm{M} \mathrm{H}_{2} \mathrm{O}_{2}$ for $12 \mathrm{~h}$ were lysed for immunoprecipitation using anti GSH and protein G beads. The immunoprecipitates were detected using the indicated antibody. ${ }^{*} P<0.05,{ }^{* *} P<0.01,{ }^{* * *} P<0.001$. Student's-t tests were used for the statistical analysis.

induce metabolic remodeling and a shift from glycolysis to the PPP, resulting in increased NADPH production, a stabilization of the redox balance and enhanced ROS clearance. In line with this finding, ENO1 and ENO2, two key enzymes involved in glycolysis where they synthesize pyruvate from D-glyceraldehyde 3-phosphate, were found to be downregulated, implying that Grx1 modulates glycolytic activity via distinct mechanisms.

\section{Grx1-mediated Modification via Deglutathionylation Might Control Specific Signaling and Affect Protein Stability}

To verify whether Grx1 regulates these proteins at the transcriptional or protein level, qRT-PCR analyses were performed to evaluate the mRNA levels when Grx1 expression was forced. We found clear evidence that the mRNA levels of SOD1, SOD2, CAT, RUVBL1, DDB1, and caveolin-1 were significantly increased, whereas the expression of PKM2, CDC25C, HSP90B1 


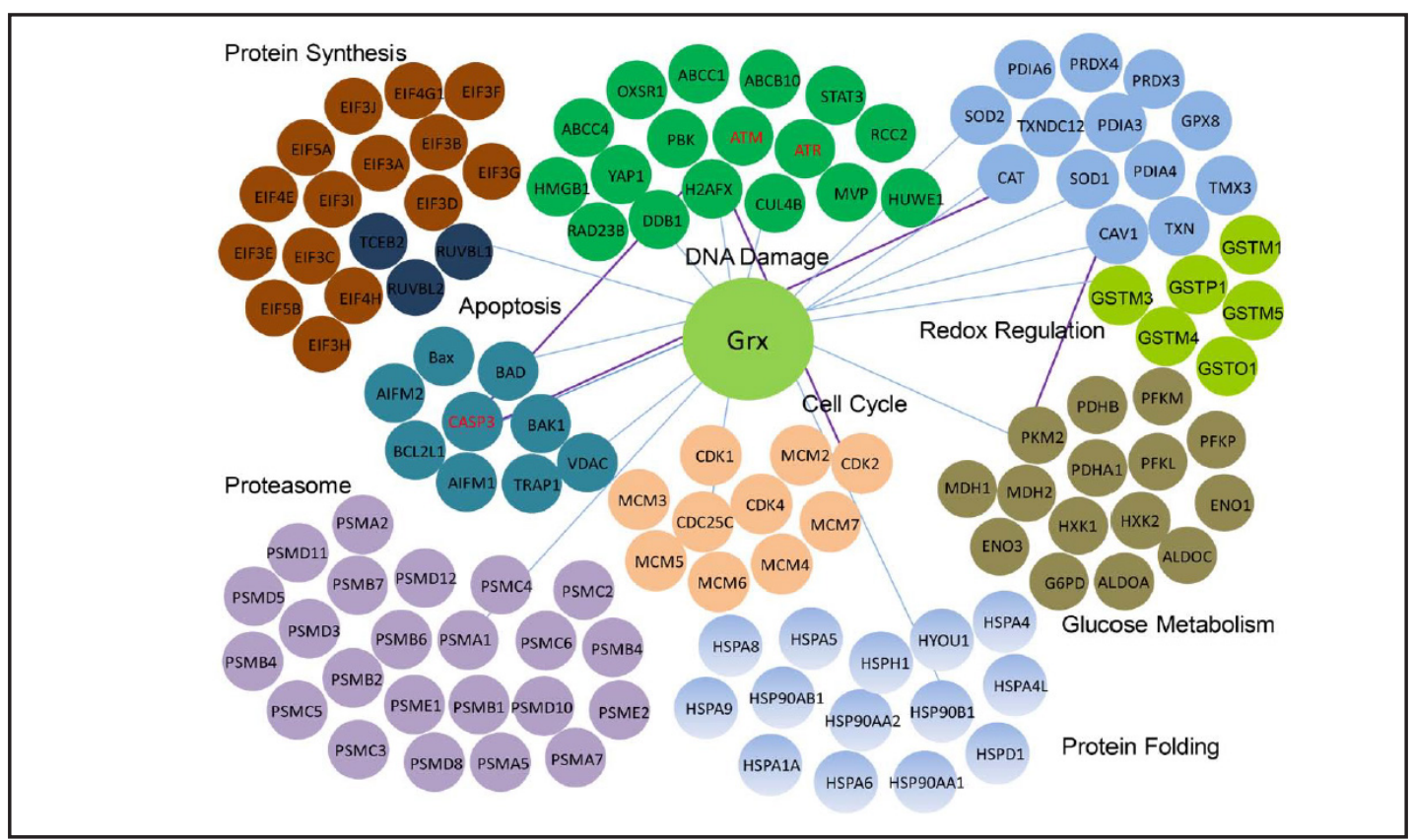

Fig. 6. Data-dependent reconstructed network showing how the signaling pathways are coordinated. The identified DEPs were submitted to DAVID and STRING for the network reconstruction, with modifications performed using Cytoscape and PowerPoint software. Validated key signaling molecules are shown with links to Grx1. Known interactions or correlations are indicated with purple lines. Novel proteins identified in this study are indicated with blue lines. Proteins unidentified in this study are displayed in red.

and VDAC1 were not obviously changed (Fig. 5A), indicating that Grx1 might regulate the stability of these proteins. We then tested for potential interactions between Grx1 and these proteins. Interestingly, we found that Grx1 selectively interacted with SOD1, SOD2, PKM2, CDC25C, HSP90B1 and VDAC1 (Fig. 5 B), which were also enriched via immunoprecipitation using an anti-GSH antibody (Fig. 5C), suggesting that deglutathionylation by Grx1 might target certain signaling proteins as a mechanism for controlling protein stability. Consistently, most of the proteins identified here have been reported to be oxidized in the presence of excessive ROS levels of [46-50]. Therefore, deglutathionylation might represent the dominant posttranslational modification (PTM) in the antioxidant response, with Grx1 playing a critical role. Overall, our study identified and confirmed several novel Grx1 substrates and highlights a crucial role of the Grx-mediated deglutathionylation-dependent regulation of protein turnover.

Data-dependent Reconstructed Network Analysis Revealed Multiple-pathway Cross-talk that Coordinates Specific Signaling and Transcriptional Mechanisms in the Antioxidant Response to $\mathrm{H}_{2} \mathrm{O}_{2}$

Given that our data show that Grx1 induces multiple signaling cascades, both transcriptionally and translationally, we next determined how this signaling cross-talk is coordinated and integrated. Using our dataset for a data-dependent network reconstruction and analysis using STRING (http://string.embl.de/), we obtained global functional links among these signals, including redox regulation, apoptosis, cell cycle control, metabolism, transcription factors, and DNA damage response (Fig. 6). This analysis helps show how Grx1 integrates and coordinates specific signaling and translation mechanisms in an interconnected, enabling a systematic response to antioxidant stress. In this global regulatory network, a number of key subnetworks, or pathway modules, were found to be interconnected to a variety of biological processes. For example, Grx1 negatively modulated numerous pro-apoptosis factors, including BAX, BAD, and BAK1. Caspase 3, a key effector protein involved in apoptotic signaling, was not identified in our dataset. However, it has 
been reported that Grx interact with and inhibit caspase-3 cleavage in a redox-dependent manner [51], suggesting that Grx is a central regulator of oxidative stress-induced apoptosis. However, catalase 1 also regulates the apoptotic pathway by interacting with caspase-3, indicating that Grx1 could integrate apoptosis with red ox regulation, resulting in an enhanced antioxidant response. Notably, several proteins involved in glycolysis were identified and found to be downregulated, including PKM2, which is also repressed by caveolin-1, resulting in decreased glycolytic activity $[52,53]$. Caveolin-1 was significantly upregulated by Grx1 upon $\mathrm{H}_{2} \mathrm{O}_{2}$ treatment, further supporting the idea that Grx1 coordinates specific signaling pathways in the response to oxidative stress.

\section{Discussion}

Age-related cataracts are the most frequent cause of treatable blindness in the world [3], and oxidative insult appears to be the most important risk factor for their formation [6]. Environmental insults, such as oxidative stress and ultraviolet radiation, are mitigated by epithelial cells through multiple cellular defense mechanisms that begin with altered gene expression. Grx1 is one of the oxidation defense systems of the lens. It has been proven that Grx1 is involved in signal transduction through the targeting of numerous crucial components of many signaling pathways [27-29]. However, the functions of Grx1 and its regulatory mechanisms are poorly understood.

In this study, we used a SILAC-based quantitative proteomic approach to perform a genome-wide identification of Grx1 target proteins and/or genes. A total of 1,291 proteins were identified as being significantly enriched $(P<0.05)$. In assessing subcellular localization, mitochondrial proteins were found to be highly enriched, suggesting that mitochondrial function is tightly linked to a Grx1-mediated antioxidant response. Based on our bioinformatics analysis, the DEPs were functionally categorized into several signaling pathways, including redox regulation, apoptosis, cell cycle control, glucose metabolism, protein synthesis, DNA damage response, protein folding, and proteasome $(P<0.001)$.

Notably, signaling pathways involved in glucose metabolism and proteasome were identified as being regulated by Grx1 for the first time in this study. We further found that Grx1 could alter glucose metabolism by reducing the expression of PKM2. PKM2 is one of the pyruvate kinase isoforms, which is responsible for catalyzing phosphoenolpyruvic acid and ADP to pyruvate and ATP [54]. It is a key enzyme in allowing the diversion of glucose6-phosphate into the PPP. Apart from its role in regulating glycolytic metabolism, PKM2 has been shown to play a role in the epigenetic control of gene transcription [55] and in promoting cell cycle progression [56]. PKM2 also been reported to be involved in multiple myeloma cell proliferation, adhesion and chemoresistance [57]. It can interact with DNA damage-binding protein 2 and reduces cell survival upon UV irradiation $[58,53]$. The overexpression of PKM2 has been detected in a various types of cancer, where it has been proven to contribute to the Warburg effect, a hallmark of cancer [59]. The direct oxidation of PKM2 at Cys358 inhibits its catalytic activity $[45,60]$, which allows for the synthesis of NADPH and reduced glutathione for ROS detoxification. However, the way in which Grx1 modulates PKM2 expression remains to be investigated. We provided further evidence supporting the idea that Grx1 either transactivates gene expression or selectively interacts with proteins such as SOD1, SOD2, VDAC1, PKM2, HSP90B1 and CDC25C. Additionally, these proteins could indeed be glutathionylated, as demonstrated by the immunoprecipitation and western blot analyses. Overall, these data imply that Grx1 interacts with and regulates the levels of SOD1, SOD2, VDAC1, PKM2, HSP90B1 and CDC25C via deglutathionylation modification. VDAC plays regulatory roles in controlling changes in mitochondrial permeability and is susceptible to oxidative stress. It has also been found in the murine neuron cell line Neuro2a that the loss of Grx1 increases the oxidation of VDAC and mitochondrial dysfunction, suggesting that the deglutathionylation of VDAC by Grx1 may protect mitochondrial function [61]. Two conserved cysteine residues of VDAC1, Cys 127 and Cys 232, are suggested to be the 
putative oxidation sites. Additionally, CDC25C was found to be reduced by $\mathrm{H} 2 \mathrm{O} 2$ stimulation. Mutations of the active-site cysteines at positions 377 and 330 have been shown to confer resistance to oxidative stress-induced degradation [48]. Although the specific cysteine(s) of VDAC1 or CDC25C that are glutathionylated by Grx1 are not yet known, the present study provides a basis and potential explanation for the deglutathionylation-dependent regulation of protein stability. Future work should focus on the underlying mechanism by which these proteins are modulated.

In addition, numerous subunits of the proteasome complex were identified to be putative targets of Grx1, suggesting that the proteasome complex might play a vital role in the Grx1-mediated antioxidant response. The ubiquitin-proteasome system (UPS), which is responsible for the degradation of $90 \%$ of all proteins in eukaryotic cells, plays an important role in protein metabolism and protein quality control [62]. Proteasome function shapes innate and adaptive immune responses [63], and the introduction of exogenous proteasomes significantly promotes cell survival and protection against the proteotoxic stress caused by ROS [64]. Indeed, an increasing numbers of studies have established the pivotal role of the proteasome complex-dependent degradation of oxidized proteins during oxidative attacks [65]. The 20S proteasome subunit is the central core of the proteasome; it binds one or two regulatory $19 \mathrm{~S}$ complexes to form the $26 \mathrm{~S}$ proteasome [66]. The $20 \mathrm{~S}$ and $26 \mathrm{~S}$ proteasomes respond differently to oxidants: the $26 \mathrm{~S}$ proteasome is much more sensitive to oxidative stress than its $20 \mathrm{~S}$ core $[67,68]$. In our dataset, most of the proteasome subunits identified were $26 \mathrm{~S}$ subunits, implying that Grx1 might specifically prevent the degradation of $26 \mathrm{~S}$ subunits induced by oxidative stress. Future studies should verify how the $26 \mathrm{~S}$ subunits are modulated and identify the biological effects of the Grx1-mediated upregulation of $26 \mathrm{~S}$ subunits and their substrates.

In this study, we performed a data-dependent quantitative proteomic analysis to identify signaling and regulatory networks that systematically operate in the Grx1-mediated antioxidant response. Some key subnetwork modules were found to be interconnected, coordinating specific signaling in the systematic response to $\mathrm{H}_{2} \mathrm{O}_{2}$.

\section{Conclusion}

Taken together, our data provide a proteome-wide identification of putative targets of Grx1 and elucidate its regulatory networks. This study will provide a basis for further mechanistic investigations.

\section{Acknowledgements}

We would like to thank all of the participants and the staff at the EENT Biobank for their valuable contributions to this research. This work was supported by the National Natural Science Foundation of China (NSFC, Grant No. 81270989) and International Science and Technology Cooperation Foundation of Shanghai (Grant No. 14430721100).

\section{Disclosure Statement}

The authors declare no conflicts of interest.

\section{References}

1 Li YJ, Jiang Q, Cao GF, Yao J, Yan B: Repertoires of autophagy in the pathogenesis of ocular diseases. Cell Physiol Biochem 2015;35:1663-1676. 


\section{Cellular Physiology Cell Physiol Biochem 2016;39:1813-1826 \begin{tabular}{l|l|l|l|l|l}
\hline DOI: 10.1159/000447881 & $\begin{array}{l}\text { () 2016 The Author(s). Published by S. Karger AG, Basel } \\
\text { www.karger.com/cpb }\end{array}$
\end{tabular} \\ Fan et al.: Grx Antioxidant Response Network}

2 Richter GM, Chung J, Azen SP, Varma R, Los Angeles Latino Eye Study Group: Prevalence of visually significant cataract and factors associated with unmet need for cataract surgery: Los Angeles Latino Eye Study. Ophthalmology 2009;116:2327-2335.

3 Congdon NG, Friedman DS, Lietman T: Important causes of visual impairment in the world today. JAMA 2003;290:2057-2060.

4 Brian G, Taylor H: Cataract blindness--challenges for the 21st century. Bull World Health Organ 2001;79:249-256.

5 Kupfer C: On presentation of the Friedenwald Award of the Association for Research in Vision and Ophthalmology to Dr. Joram Piatigorsky. Invest Ophthalmol Vis Sci 1987;28:2-8.

6 Beebe DC, Holekamp NM, Shui YB: Oxidative damage and the prevention of age-related cataracts. Ophthalmic Res 2010;44:155-165.

$7 \quad$ Zheng Y, Liu Y, Ge J, Wang X, Liu L, Bu Z, Liu P: Resveratrol protects human lens epithelial cells against H2O2-induced oxidative stress by increasing catalase, SOD-1, and HO-1 expression. Mol Vis 2010;16:14671474.

8 Raghavachari N, Krysan K, Xing K, Lou MF: Regulation of thioltransferase expression in human lens epithelial cells. Invest Ophthalmol Vis Sci 2001;42:1002-1008.

9 Prinarakis E, Chantzoura E, Thanos D, Spyrou G: S-glutathionylation of IRF3 regulates IRF3-CBP interaction and activation of the IFN beta pathway. EMBO J 2008;27:865-875.

10 Löfgren S, Fernando MR, Xing KY, Wang Y, Kuszynski CA, Ho YS, Lou MF: Effect of thioltransferase (glutaredoxin) deletion on cellular sensitivity to oxidative stress and cell proliferation in lens epithelial cells of thioltransferase knockout mouse. Invest Ophthalmol Vis Sci 2008;49:4497-4505.

11 Pai HV, Starke DW, Lesnefsky EJ, Hoppel CL, Mieyal JJ: What is the functional significance of the unique location of glutaredoxin 1 (GRx1) in the intermembrane space of mitochondria? Antioxid Redox Signal 2007;9:2027-2033.

12 Gao XH, Qanungo S, Pai HV, Starke DW, Steller KM, Fujioka H, Lesnefsky EJ, Kerner J, Rosca MG, Hoppel CL, Mieyal JJ: Aging-dependent changes in rat heart mitochondrial glutaredoxins--Implications for redox regulation. Redox Biol 2013;1:586-598.

13 Holmgren A: Thioredoxin and glutaredoxin systems. J Biol Chem 1989;264:13963-13966.

14 Chartoumpekis DV, Ziros PG, Sykiotis GP, Zaravinos A, Psyrogiannis AI, Kyriazopoulou VE, Spandidos DA, Habeos IG: Nrf2 activation diminishes during adipocyte differentiation of ST2 cells. Int J Mol Med 2011;28:823-828.

15 Kalinina EV, Chernov NN, Saprin AN: Involvement of thio-, peroxi-, and glutaredoxins in cellular redoxdependent processes. Biochemistry (Mosc) 2008;73:1493-1510.

16 Zahedi Avval F, Holmgren A: Molecular mechanisms of thioredoxin and glutaredoxin as hydrogen donors for Mammalian s phase ribonucleotide reductase. J Biol Chem 2009;284:8233-8240.

17 Reynaert NL, van d V, Guala AS, McGovern T, Hristova M, Pantano C, Heintz NH, Heim J, Ho YS, Matthews DE, Wouters EF, Janssen-Heininger YM: Dynamic redox control of NF-kappaB through glutaredoxin-regulated S-glutathionylation of inhibitory kappaB kinase beta. Proc Natl Acad Sci U S A 2006;103:13086-13091.

18 Inadomi C, Murata H, Ihara Y, Goto S, Urata Y, Yodoi J, Kondo T, Sumikawa K: Overexpression of glutaredoxin protects cardiomyocytes against nitric oxide-induced apoptosis with suppressing the S-nitrosylation of proteins and nuclear translocation of GAPDH. Biochem Biophys Res Commun 2012;425:656-661.

19 Li S, Sun Y, Qi X, Shi Y, Gao H, Wu Q Liu X, Yu H, Zhang C: Protective effect and mechanism of glutaredoxin 1 on coronary arteries endothelial cells damage induced by high glucose. Biomed Mater Eng 2014;24:38973903.

20 Cater MA, Materia S, Xiao Z, Wolyniec K, Ackland SM, Yap YW, Cheung NS, La Fontaine S: Glutaredoxin1 protects neuronal cells from copper-induced toxicity. Biometals 2014;27:661-672.

21 Lillig CH, Berndt C, Vergnolle O, Lonn ME, Hudemann C, Bill E, Holmgren A: Characterization of human glutaredoxin 2 as iron-sulfur protein: A possible role as redox sensor. Proc Natl Acad Sci U S A 2005;102:8168-8173.

22 Enoksson ME, Fernandes AP, Prast S, Lillig CH, Holmgren A, Orrenius S: Overexpression of glutaredoxin 2 attenuates apoptosis by preventing cytochrome c release. Biochem Biophys Res Commun 2005;327:774779.

23 Wu H, Xing K, Lou MF: Glutaredoxin 2 prevents H(2)O(2)-induced cell apoptosis by protecting complex I activity in the mitochondria. Biochim Biophys Acta 2010;1797:1705-1715. 


\section{Cellular Physiology Cell Physiol Biochem 2016;39:1813-1826 \begin{tabular}{l|l|l|}
\hline DOI: 10.1159/000447881 & $\begin{array}{l}\text { C) 2016 The Author(s). Published by S. Karger AG, Basel } \\
\text { www.karger.com/cpb }\end{array}$ \\
\hline Published online: October 17, 2016 Biochemistry
\end{tabular} \\ Fan et al.: Grx Antioxidant Response Network}

24 Zhang J, Yan H, Löfgren S, Tian X, Lou MF: Ultraviolet radiation-induced cataract in mice: the effect of age and the potential biochemical mechanism. Invest Ophthalmol Vis Sci 2012;53:7276-7285.

25 Meyer LM, Löfgren S, Ho YS, Lou M, Wegener A, Holz F, Söderberg P: Absence of glutaredoxin1 increases lens susceptibility to oxidative stress induced by UVR-B. Exp Eye Res 2009;89:833-839.

26 Kronschläger M, Galichanin K, Ekström J, Lou MF, Söderberg PG: Protective effect of the thioltransferase gene on in vivo UVR-300 nm-induced cataract. Invest Ophthalmol Vis Sci 2012;53:248-252.

27 Song JJ, Lee YJ: Differential role of glutaredoxin and thioredoxin in metabolic oxidative stress-induced activation of apoptosis signal-regulating kinase 1. Biochem J 2003;373:845-853.

28 Chantzoura E, Prinarakis E, Panagopoulos D, Mosialos G, Spyrou G: Glutaredoxin-1 regulates TRAF6 activation and the IL-1 receptor/TLR4 signalling. Biochem Biophys Res Commun 2010;403:335-339.

29 Liu X, Jann J, Xavier C, Wu H: Glutaredoxin 1 (Grx1) protects human retinal pigment epithelial cells from oxidative damage by preventing AKT glutathionylation. Invest Ophthalmol Vis Sci 2015;56:2821-2832.

30 Ong SE, Blagoev B, Kratchmarova I, Kristensen DB, Steen H, Pandey A, Mann M: Stable isotope labeling by amino acids in cell culture, SILAC, as a simple and accurate approach to expression proteomics. Mol Cell Proteomics 2002;1:376-386.

31 Ong See, Mann M: A practical recipe for stable isotope labeling by amino acids in cell culture (SILAC). Nat Protoc 2006;1:2650-2660.

32 Zhang Y, Liu Y, Duan J, Yan H, Zhang J, Zhang H, Fan Q, Luo F, Yan G, Qiao K, Liu J: Hippocalcin-like 1 suppresses hepatocellular carcinoma progression by promoting p21Waf/Cip1 stabilization via activating ERK1/2-MAPK pathway. Hepatology 2016;63:880-897.

33 Du R, Long J, Yao J, Dong Y, Yang X, Tang S, Zuo S, He Y, Chen X: Subcellular quantitative proteomics reveals multiple pathway cross-talk that coordinates specific signaling and transcriptional regulation for the early host response to LPS. J Proteome Res 2010;9:1805-1821.

34 Tang S, Bai C, Yang P, Chen X: 14-3-3ع boosts bleomycin-induced DNA damage response by inhibiting the drug-resistant activity of MVP. J Proteome Res 2013;12:2511-2524.

35 Kreuz S, Fischle W: Oxidative stress signaling to chromatin in health and disease. Epigenomics 2016;8:843862.

36 Kezic A, Spasojevic I, Lezaic V, Bajcetic M: Mitochondria-targeted antioxidants: future perspectives in kidney ischemia Reperfusion injury.Oxid Med Cell Longev 2016;2016:2950503.

37 Solesio ME, Prime TA, Logan A, Murphy MP, Del Mar Arroyo-Jimenez M, Jordán J, Galindo MF: The mitochondria-targeted anti-oxidant MitoQ reduces aspects of mitochondrial fission in the 6-OHDA cell model of Parkinson's disease. Biochim Biophys Acta 2013;1832:174-182.

38 Phaniendra A, Jestadi DB, Periyasamy L: Free radicals: properties, sources, targets, and their implication in various diseases. Indian J Clin Biochem 2015;30:11-26.

39 Kriegenburg F, Poulsen EG, Koch A, Krüger E, Hartmann-Petersen R: Redox control of the ubiquitinproteasome system: from molecular mechanisms to functional significance. Antioxid Redox Signal 2011;15:2265-2299.

40 Raynes R, Pomatto LCD, Davies KJA: Degradation of oxidized proteins by the proteasome: distinguishing between the 20S, 26S, and immunoproteasome proteolytic pathways. Mol Aspects Med DOI: 10.1016/j. mam.2016.05.001.

41 Wang P, Peng X, Wei ZF, Wei FY, Wang W, Ma WD, Yao LP, Fu YJ, Zu YG: Geraniin exerts cytoprotective effect against cellular oxidative stress by upregulation of Nrf2-mediated antioxidant enzyme expression via PI3K/AKT and ERK1/2 pathway. Biochim Biophys Acta 2015;1850:1751-1761.

42 Park JH, Choi JW, Ju EJ, Pae AN, Park KD: Antioxidant and anti-inflammatory activities of a natural Compound, Shizukahenriol, through Nrf2 activation. Molecules 2015;20:15989-16003.

43 Bhattacharya A, Hegazy AN, Deigendesch N, Kosack L, Cupovic J, Kandasamy RK, Hildebrandt A, Merkler D, Kühl AA, Vilagos B, Schliehe C, Panse I, Khamina K, Baazim H, Arnold I, Flatz L, Xu HC, Lang PA, Aderem A, Takaoka A, Superti-Furga G, Colinge J, Ludewig B, Löhning M, Bergthaler A: Superoxide dismutase 1 protects hepatocytes from Type I interferon-driven oxidative damage. Immunity 2015;43:974-986.

44 Tsang CK, Liu Y, Thomas J, Zhang Y, Zheng XF: Superoxide dismutase 1 acts as a nuclear transcription factor to regulate oxidative stress resistance. Nat Commun 2014;5:3446.

45 Anastasiou D, Poulogiannis G, Asara JM, Boxer MB, Jiang JK, Shen M, Bellinger G, Sasaki AT, Locasale JW, Auld DS, Thomas CJ, Vander Heiden MG, Cantley LC: Inhibition of pyruvate kinase M2 by reactive oxygen species contributes to cellular antioxidant responses. Science 2011;334:1278-1283. 


\section{Cellular Physiology Cell Physiol Biochem 2016;39:1813-1826 \begin{tabular}{l|l|l|l|} 
DOI: 10.1159/000447881 & $\begin{array}{l}\text { () 2016 The Author(s). Published by S. Karger AG, Basel } \\
\text { www.karger.com/cpb }\end{array}$
\end{tabular} \\ Fan et al.: Grx Antioxidant Response Network}

46 Cohen RA, Murdoch CE, Watanabe Y, Bolotina VM, Evangelista AM, Haeussler DJ, Smith MD, Mei Y, Tong X, Han J, Behring JB, Bachschmid MM, Matsui R: Endothelial cell redox regulation of ischemic angiogenesis.J Cardiovasc Pharmacol 2016;67:458-464.

47 Sabens Liedhegner EA, Gao XH, Mieyal JJ: Mechanisms of altered redox regulation in neurodegenerative diseases--focus on S--glutathionylation. Antioxid Redox Signal 2012;16:543-566.

48 Savitsky PA, Finkel T: Redox regulation of Cdc25C. J Biol Chem 2002;277:20535-20540.

49 Watanabe Y, Cohen RA, Matsui R: Redox regulation of ischemic angiogenesis-another aspect of reactive oxygen species. Circ J 2016;80:1278-1284.

50 McAlary L, Yerbury JJ, Aquilina JA: Glutathionylation potentiates benign superoxide dismutase 1 variants to the toxic forms associated with amyotrophic lateral sclerosis. Sci Rep 2013;3:3275.

51 Pan S, Berk BC: Glutathiolation regulates tumor necrosis factor-alpha-induced caspase-3 cleavage and apoptosis: key role for glutaredoxin in the death pathway. Circ Res 2007;100:213-219.

52 Chiavarina B, Whitaker-Menezes D, Martinez-Outschoorn UE, Witkiewicz AK, Birbe R, Howell A, Pestell RG, Smith J, Daniel R, Sotgia F, Lisanti MP: Pyruvate kinase expression (PKM1 and PKM2) in cancer-associated fibroblasts drives stromal nutrient production and tumor growth. Cancer Biol Ther 2011;12:1101-1113.

53 Bonuccelli G, Whitaker-Menezes D, Castello-Cros R, Pavlides S, Pestell RG, Fatatis A, Witkiewicz AK, Vander Heiden MG, Migneco G, Chiavarina B, Frank PG, Capozza F, Flomenberg N, Martinez-Outschoorn UE, Sotgia F, Lisanti MP: The reverse Warburg effect: glycolysis inhibitors prevent the tumor promoting effects of caveolin-1 deficient cancer associated fibroblasts. Cell Cycle 2010;9:1960-1971.

54 Israelsen WJ, Vander Heiden MG: Pyruvate kinase: function, regulation and role in cancer. Semin Cell Dev Biol 2015;43:43-51.

55 Yang W, Xia Y, Hawke D, Li X, Liang J, Xing D, Aldape K, Hunter T, Alfred Yung WK, Lu Z: PKM2 phosphorylates histone $\mathrm{H} 3$ and promotes gene transcription and tumorigenesis. Cell 2012;150:685-696.

56 Jiang Y, Li X, Yang W, Hawke DH, Zheng Y, Xia Y, Aldape K, Wei C, Guo F, Chen Y, Lu Z: PKM2 regulates chromosome segregation and mitosis progression of tumor cells. Mol Cell 2014;53:75-87.

57 He Y, Wang Y, Liu H, Xu X, He S, Tang J, Huang Y, Miao X, Wu Y, Wang Q, Cheng C: Pyruvate kinase isoform M2 (Pkm2) participates in multiple myeloma cell proliferation, adhesion and chemoresistance. Leuk Res 2015;39:1428-1436.

58 Xie X, Wang M, Mei J, Hu F, Ding F, Lv L: Pyruvate kinase M2 interacts with DNA damage-binding protein 2 and reduces cell survival upon UV irradiation. Biochem Biophys Res Commun 2015;467:427-433.

59 Hu W, Lu SX, Li M, Zhang C, Liu LL, Fu J, Jin JT, Luo RZ, Zhang CZ, Yun JP: Pyruvate kinase M2 prevents apoptosis via modulating Bim stability and associates with poor outcome in hepatocellular carcinoma. Oncotarget 2015;6:6570-6583.

60 Kuehne A, Emmert H, Soehle J, Winnefeld M, Fischer F, Wenck H, Gallinat S, Terstegen L, Lucius R, Hildebrand J, Zamboni N: Acute activation of oxidative pentose phosphate pathway as first-line response to oxidative stress in human skin cells. Mol Cell 2015;59:359-371.

61 Saeed U, Durgadoss L, Valli RK, Joshi DC, Joshi PG, Ravindranath V: Knockdown of cytosolic glutaredoxin 1 leads to loss of mitochondrial membrane potential: implication in neurodegenerative diseases. PLoS One 2008;3:e2459.

62 Wang F, Lerman A, Herrmann J: Dysfunction of the ubiquitin-proteasome system in atherosclerotic cardiovascular disease. Am J Cardiovasc Dis 2015;5:83-100.

63 Kammerl IE, Meiners S: Proteasome function shapes innate and adaptive immune responses. Am J Physiol Lung Cell Mol Physiol DOI: 10.1152/ajplung.00156.2016.

64 Han DH, Na HK, Choi WH, Lee JH, Kim YK, Won C, Lee SH, Kim KP, Kuret J, Min DH, Lee MJ: Direct cellular delivery of human proteasomes to delay tau aggregation. Nat Commun 2014;5:5633.

65 Catalgol B, Grune T: Protein pool maintenance during oxidative stress. Curr Pharm Des 2009;15:30433051.

66 Peters JM, Cejka Z, Harris JR, Kleinschmidt JA, Baumeister W: Structural features of the 26S proteasome complex. J Mol Biol 1993;234:932-937.

67 Wang X, Yen J, Kaiser P, Huang L: Regulation of the 26S proteasome complex during oxidative stress. Sci Signal 2010;7:ra88. DOI: 10.1126/scisignal.2001232.

68 Aiken CT, Kaake RM, Wang X, Huang L: Oxidative stress-mediated regulation of proteasome complexes. Mol Cell Proteomics 2011;10:R110.006924. DOI: 10.1074/mcp.M110.006924. 